\title{
Hair Shedding Evaluation for Alopecia: A Refined Wash Test
}

\author{
Xiangqian Li D, Xianghe Wang, Chen Wang, Jianzhong Zhang, Cheng Zhou \\ Department of Dermatology, Peking University People's Hospital, Beijing, People's Republic of China
}

Correspondence: Cheng Zhou, Department of Dermatology, Peking University People's Hospital, No. II Xizhimen South Street, Xicheng District, Beijing, 100044, People's Republic of China, Email chengzhou@live.cn

\begin{abstract}
Purpose: The aim of this study is to establish a refined wash test (RWT) and explore the characteristics of daily shedding hair parameters in normal individuals and patients with androgenetic alopecia (AGA) or telogen effluvium (TE).

Materials and Methods: Chinese subjects diagnosed as normal, AGA or TE were enrolled. For the RWT procedure, the subjects were asked to pre-wash their hair 24 hours before the first collection, then to wash and rinse their hair with flowing water above a sink covered with a piece of filtering cloth. All hairs entrapped in the filtering cloth were air dried and collected. This "wash-and-collect" process was repeated for 3 consecutive days with a $24 \pm 2$ hour interval. Collected hairs were counted manually, and hair diameter was measured by videodermoscope. Number and diameter of daily shedding hairs and percentage of vellus and terminal hairs were analyzed.

Results: A total of 176 subjects participated in and completed the study. Shedding hair number during daily shampooing was $27.9 \pm$ $12.2,52.2 \pm 28.5$ and $125.5 \pm 62.7$ hairs in normal, AGA and TE participants, respectively, and the differences between each two of the three groups were all statistically significant. Diameter of shedding hairs was $76.9 \pm 9.0 \mu \mathrm{m}, 60.0 \pm 9.5 \mu \mathrm{m}$ and $82.7 \pm 9.2 \mu \mathrm{m}$ in normal, AGA and TE, respectively. Along with advancement of AGA severity, shedding hair number was generally increased, while hair diameter decreased. The percentage of vellus hairs in AGA was significantly higher than in the normal and TE groups $(8.3 \pm 6.6 \%$ vs $1.0 \pm 1.6 \%$ and $1.0 \pm 1.0 \%$ ). The combination of shedding hair number and percentage of vellus hairs had a high diagnostic confidence $(\mathrm{AUC}=0.957)$ to diagnose AGA from normal. Shedding hair number alone showed high confidence to diagnose TE from normal (AUC $=0.999)$.
\end{abstract}

Conclusion: The non-invasive, easy-to-use RWT established in this study is a valuable tool to evaluate daily shedding hairs, which may be useful in diagnosis, dynamic monitoring of disease progression and treatment efficacy.

Keywords: hair loss, hair shedding, refined wash test, androgenetic alopecia, telogen effluvium

\section{Introduction}

Hair loss disorders or alopecia are common in dermatology practice and cause significant impacts on patients' quality of life, reduce self-esteem, and increase stress. ${ }^{1,2}$ The basic categories of alopecia include both cicatricial (such as lichen planopilaris, discoid lupus erythematosus and folliculitis decalvans) and non-cicatricial alopecias. ${ }^{3}$ Androgenetic alopecia (AGA) and telogen effluvium (TE) are two of the most common causes of non-cicatricial alopecia. ${ }^{3}$ Although the diagnoses and distinctions of AGA and TE are not difficult in typical situations, atypical cases (such as alopecia with atypical distributions, initial stage of AGA or chronic TE) are troublesome. ${ }^{4}$ In addition, accurately assessing the improvement or aggravation of hair loss is important for evaluation of disease severity and management.

So far, the evaluation methods of hair loss or growth are generally divided into three categories: non-invasive, semiinvasive and invasive ways. ${ }^{5}$ Non-invasive methods include hair weight, hair counts, pull test, wash test, global photography, dermoscopy and phototrichogram. ${ }^{5,6}$ Semi-invasive technique comprises trichogram. ${ }^{5,7}$ Scalp biopsy is the main invasive method. ${ }^{5}$

For most patients with hair loss, the main complaint is increased daily hair shedding, especially during hair washing. ${ }^{8}$ The everyday shedding hairs, which may contain tremendous diagnostic information, are probably underestimated, and 
we believed that they were just like hidden treasure that needs further exploration. As one of the non-invasive methods for hair shedding evaluation, wash test has long been introduced but underutilized in practice.

In 1999, the wash test was first described by Rampini et al, ${ }^{9}$ which required prepubertal subjects to soap and rinse their hair in a sink with the hole covered by gauze after 5 days abstinence from shampooing. The authors received a valid estimate value of "normalcy" with $10.68 \pm 3.91$ hairs. $^{9}$ In 2005, a modified wash test (MWT) was developed by Rebora et $\mathrm{al}^{10}$ for diagnosing AGA and chronic TE. The method was designed based on the points that shedding hairs in AGA patients will be shorter in length (vellus hairs have their own short anagen phase) and less in number than those in TE patients during hair washing. ${ }^{10}$ Patients shedding $\leq 100$ hairs and with $\geq 10 \%$ vellus hair ( $\leq 3 \mathrm{~cm}$ in length) were diagnosed as AGA, those shedding $>100$ hairs and with $<10 \%$ vellus hair as TE, and those shedding $>100$ hairs and with $\geq 10 \%$ vellus hair as AGA combined with TE. ${ }^{10}$ The reliability of MWT was evaluated and verified in another study. ${ }^{11}$ However, the MWT has not been widely adopted because patients are required to avoid shampooing for 5 days, it is not suitable for patients with hairs shorter than $3 \mathrm{~cm}$, and it provides little information regarding daily shed.

It is usual to mention that humans shed between 50 and 100 scalp hairs per day; however, it is extremely difficult to collect all shedding hairs during daily activities. Though hair shedding is most significant during hair washing, there are no previous studies on shedding hair numbers of daily washing. Furthermore, a standardized method that could collect everyday shedding hairs during hair washing still needs to be established.

In this study, we established and evaluated a 3-consecutive-days refined wash test (RWT) for evaluation of daily hair shedding. Characteristics of daily shedding hair collected by RWT in normal individuals and patients with AGA or TE were analyzed.

\section{Materials and Methods}

\section{Subjects}

Informed consent was obtained from 176 participants and this study was approved by the Institutional Ethics Committee of the Peking University People's Hospital, Beijing, China. The study was conducted in accordance with the Declaration of Helsinki. All subjects were Chinese Han adults enrolled from the dermatology clinics between June 2019 and October 2020. Diagnoses were performed by two independent dermatologists who had at least one year's specialist hair training.

Inclusion criteria:

(a) Male or female subject above the age of 18 years.

(b) Subject without any symptom or sign (confirmed by handheld dermoscope) of hair loss and without a current or recent history of secondary hair loss induced by systemic diseases, such as thyroid disease, anemia et al, was included as normal volunteer.

(c) Subject with the following criteria regarding to AGA must be met:

- clinical diagnosis of AGA with detailed medical history, clinical examination and dermoscopy. ${ }^{12}$

- Hamilton-Norwood classification score (male only) of II to VII, or with Ludwig scale (female or male ${ }^{13}$ ) of I to III.

(d) Subject manifested as acute or chronic diffuse telogen hair loss after the inciting factor, with positive pull test and characteristic dermoscopic features was included as TE patient. ${ }^{12,14}$

(e) Ability to comprehend and willing to sign an informed consent form.

Exclusion criteria:

(a) Subject with other non-scarring alopecia (such as alopecia areata, trichotillomania), scarring alopecia (such as lichen planopilaris, frontal fibrosing alopecia, discoid lupus erythematosus) or hair shaft disorders, was excluded.

(b) Subject with scalp condition (eg, scar, giant nevus) or systemic abnormalities that might involve hair growth and interfere with the results of the test. 
(c) Subject using any products (eg, finasteride, minoxidil, anti-androgens) or devices (eg, laser, microneedling therapy) for promoting scalp hair growth within previous 6 months.

(d) Use of medications that potentially cause drug-induced hair loss within previous 6 months.

(e) History of hair transplantation on the scalp.

(f) Hair perming or hair dyeing within previous 3 months.

\section{The Procedure of Refined Wash Test (RWT)}

In order to diminish the impact of unwashed time before the test and to ensure a uniform baseline, all participants were asked to wash their hair 24 hours before the first collection. During the RWT period, the subjects were instructed to spread a piece of filter cloth with light color over the sink, then shampoo and rinse with flowing water above a sink covered with filtering cloth. All hairs entrapped in the filtering cloth were air dried, and then collected and placed into small zip lock plastic bags labeled with name and date (Figure 1). This "wash-and-collect" process was repeated for 3 consecutive days with a $24 \pm 2$ hour interval between every two shampooings. Our prior data indicated that there was no significant difference in all parameters of daily shedding hairs in the RWT for the short term (3 consecutive days) vs long term (7 consecutive days) (Supplemental Table 1).

The following were several caveats for doing the procedure:

(a) For subjects with longer hair, not all the hairs detached from scalp could be rinsed off by the water because of the inter-hair shaft friction. Therefore the shedding hairs during the drying process with instant dry hair towel and low speed air drier right after the hair wash should also be collected and put into the bag of this day.
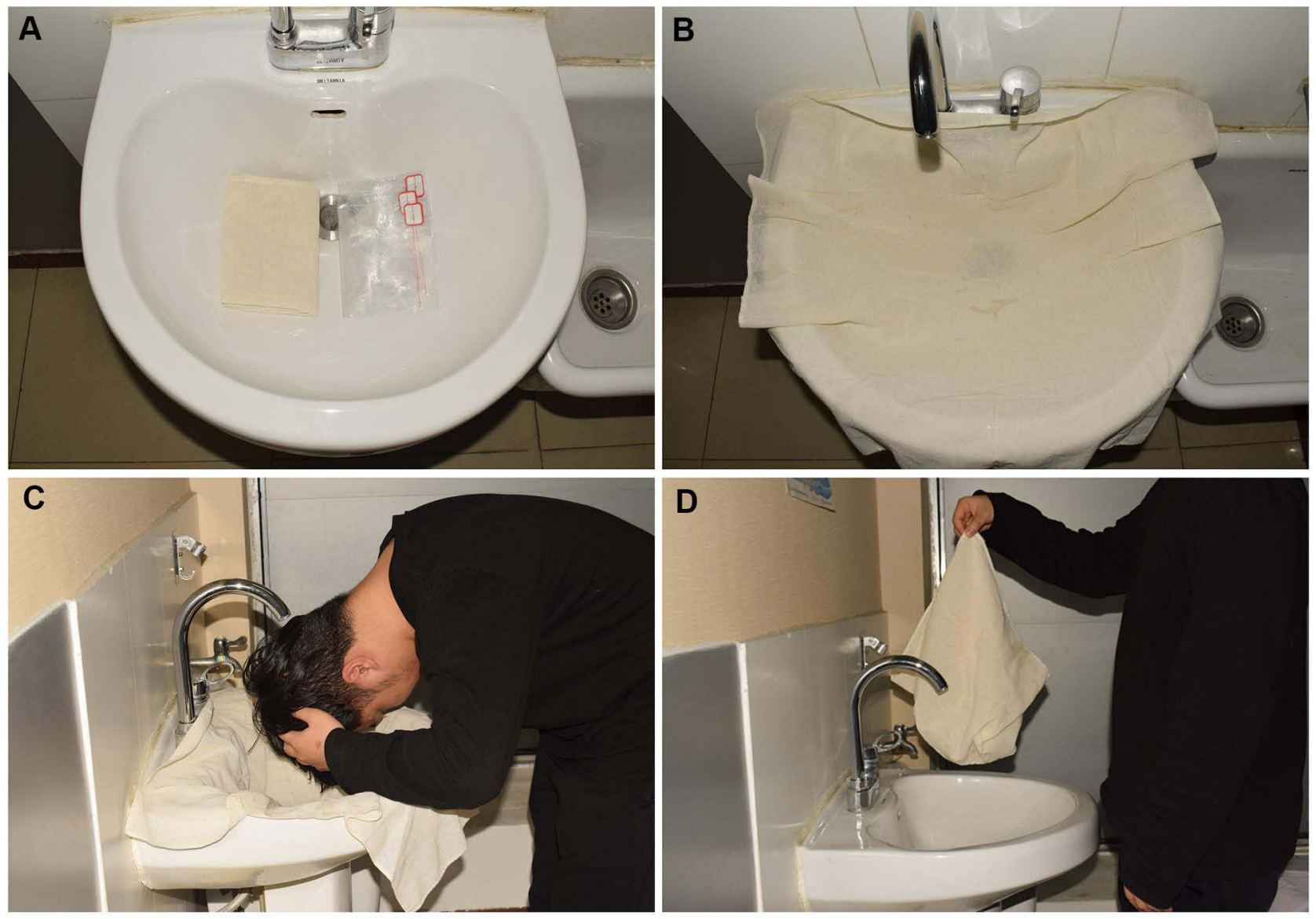

Figure I The demonstration of refined wash test procedure. (A) The supplies necessary for the test. (B-D) The subjects were asked to spread a piece of filter cloth with light color over the sink and shampoo with flowing water above the cloth. They washed their hair once, from hair roots to tips, rinsed the hairs gently and kept the water draining away directly to the sewer. 
(b) During the test, combing (with wide tooth comb or hand running through hairs) was restricted to 2 times or less per day and it should be avoided within 1 hour before washing.

(c) Only one shampoo per day was allowed, which was applied during the RWT procedure.

\section{Hair Counting and Hair Diameter Measurement}

Collected hairs were neatly pasted on scotch tape with forceps and counted by two independent investigators. Hair diameter was measured at the level of $0.5 \mathrm{~cm}$ above the hair bulb using videodermoscopy equipped with TrichoScale ${ }^{\circledR}$ (Fotofinder Systems GmbH, Bad Birnbach, Germany) at 70-fold magnification (Supplemental Figure 1). All the hairs were classified into 3 types based on different diameters: vellus hair (less than $30 \mu \mathrm{m})$, intermediate hair (30 to $60 \mu \mathrm{m})$ and terminal hair (more than $60 \mu \mathrm{m}){ }^{14,15}$

\section{Statistical Analysis}

Data characteristics were presented as mean with SDs. The Kruskal-Wallis test was used to compare the participants' age between groups. The parameters of shedding hair were conducted by paired-sample $t$-test for consistency of data on different duration and one-way analysis of variance (one-way ANOVA) for comparison between different alopecia patients and normal volunteers. Receiver operating characteristic (ROC) curves were employed to calculate area under curve (AUC) values. Diagnostic efficiency of shedding hair parameters was estimated using AUC analysis with Youden index to determine the optimal cut-off value. Statistical analyses were performed using IBM SPSS version 26.0 for Windows (IBM Corp., CA, USA). The minimal sample size was estimated by PASS (procedure: one-way analysis of variance), using the prior data. A value of $\mathrm{p}<0.05$ was considered statistically significant.

\section{Results}

\section{Participant Characteristics}

A total of 176 subjects (81 males and 95 females) were included in this study. Detailed summary of the demographic data is described in Table 1. The mean age of participants was 33.2 years, with a range from 18 to 59 years. The normal group consisted of 71 participants (22 males and 49 females) with a median age of $34.6 \pm 11.4$ years. AGA group comprised 77 patients (54 males and 23 females) with a median age of $32.0 \pm 9.5$ years. TE group comprised 28 subjects (5 males and 23 females) aged $33.8 \pm 10.2$ years. Study participants' age had no significant difference among groups $(\mathrm{H}=1.274, \mathrm{P}=$ 0.735). No subject of type VII of MPHL or type III of FPHL was successfully recruited.

\section{Number of Shedding Hairs}

The results for average number of shedding hairs were presented in Table 2 and Figure 2. Normal participants showed the lowest number of $27.9 \pm 12.2$ (mean \pm SD) hairs and the greatest number of $125.5 \pm 62.7$ hairs was observed in TE patients. Shedding hair count in AGA patients was $52.2 \pm 28.5$ hairs. The number in TE patients were significantly higher than those of the other two groups, and value in AGA patients was significantly higher than that in normal subjects $(p<0.001)$.

Table I Characteristics of Study Participants

\begin{tabular}{|c|c|c|c|c|c|c|c|c|c|}
\hline \multirow[t]{3}{*}{ Characteristic } & \multirow[t]{3}{*}{ Normal } & \multicolumn{6}{|c|}{ AGA (Varying Degrees of Baldness) } & \multirow[t]{3}{*}{ TE } & \multirow[t]{3}{*}{ Total } \\
\hline & & \multicolumn{4}{|c|}{ Hamilton-Norwood Classification } & \multicolumn{2}{|c|}{ Ludwig Classification } & & \\
\hline & & III & IV & $\mathbf{V}$ & VI & $\mathbf{I}$ & II & & \\
\hline $\begin{array}{l}\text { Participants, no. } \\
\text { Sex }\end{array}$ & 71 & 15 & 12 & 8 & 8 & 19 & 15 & 28 & 176 \\
\hline Male & 22 & 15 & 12 & 8 & 8 & 7 & 4 & 5 & 81 \\
\hline Female & 49 & 0 & 0 & 0 & 0 & 12 & 11 & 23 & 95 \\
\hline Mean age, y (SD) & 34.6 (II.4) & $28.5(8.3)$ & $32.2(5.1)$ & $29.4(6.0)$ & $50.6(9.3)$ & $26.5(4.7)$ & $34.0(7.5)$ & $33.8(10.2)$ & $33.2(10.3)$ \\
\hline
\end{tabular}

Abbreviations: AGA, androgenetic alopecia; TE, telogen effluvium. 
Table 2 Shedding Hair Parameters of the Refined Wash Test in Normal, AGA and TE Groups

\begin{tabular}{|c|c|c|c|c|}
\hline Classification & Shedding Hairs, No. & Hair Diameter $(\mu \mathrm{m})$ & Vellus Hairs (\%) & Terminal Hairs (\%) \\
\hline & Mean \pm SD & Mean \pm SMean \pm SD & Mean \pm SD & Mean \pm SD \\
\hline Normal & $27.9 \pm 12.2^{\mathrm{a}}$ & $76.9 \pm 9.0^{a}$ & $1.0 \pm 1.6^{\mathrm{a}}$ & $79.7 \pm 12.7^{\mathrm{a}}$ \\
\hline AGA & $52.2 \pm 28.5^{\mathrm{b}}$ & $60.0 \pm 9.5^{b}$ & $8.3 \pm 6.6^{b}$ & $48.8 \pm 20.1^{b}$ \\
\hline TE & $125.5 \pm 62.7^{c}$ & $82.7 \pm 9.2^{c}$ & $1.0 \pm 1.0^{\mathrm{a}}$ & $85.3 \pm 8.7^{\mathrm{a}}$ \\
\hline $\mathrm{p}$-value ${ }^{\S}$ & $<0.001 *$ & $<0.00 I^{*}$ & $<0.00 I^{*}$ & $<0.001 *$ \\
\hline Normal & $27.9 \pm 12.2^{\mathrm{a}}$ & $76.9 \pm 9.0^{\mathrm{a}}$ & $1.0 \pm 1.6^{\mathrm{a}}$ & $79.7 \pm 12.7^{\mathrm{a}}$ \\
\hline \multicolumn{5}{|l|}{ AGA } \\
\hline Male pattern & $58.8 \pm 32.7^{\mathrm{b}}$ & $57.4 \pm 8.2^{b}$ & $8.2 \pm 6.1^{b}$ & $43.4 \pm 18.3^{b}$ \\
\hline Female pattern & $43.7 \pm 19.6^{c}$ & $63.2 \pm 10.2^{c}$ & $8.3 \pm 7.2^{b}$ & $55.6 \pm 20.4^{c}$ \\
\hline $\mathrm{p}$-value ${ }^{\S}$ & $<0.00 I^{*}$ & $<0.001 *$ & $<0.001 *$ & $<0.00 I^{*}$ \\
\hline Normal & $27.9 \pm 12.2^{\mathrm{a}}$ & $76.9 \pm 9.0^{\mathrm{a}}$ & $1.0 \pm 1.6^{\mathrm{a}}$ & $79.7 \pm 12.7^{\mathrm{a}}$ \\
\hline \multicolumn{5}{|l|}{ AGA } \\
\hline Hamilton-Norwood III & $48.7 \pm 23.8^{\mathrm{b}}$ & $64.4 \pm 7.1^{\mathrm{b}}$ & $4.4 \pm 2.7^{b}$ & $57.9 \pm 15.3^{b}$ \\
\hline Hamilton-Norwood IV & $60.5 \pm 34.3^{b}$ & $56.4 \pm 5.6^{\mathrm{b}, \mathrm{c}}$ & $7.0 \pm 1.9^{b}$ & $40.6 \pm 15.4^{c}$ \\
\hline Hamilton-Norwood V & $76.3 \pm 44.6^{\mathrm{a}, \mathrm{b}}$ & $54.1 \pm 6.6^{c}$ & $12.7 \pm 8.1^{\mathrm{b}}$ & $39.9 \pm 14.3^{c}$ \\
\hline Hamilton-Norwood VI & $58.1 \pm 29.3^{a, b}$ & $49.2 \pm 3.7^{c}$ & $12.8 \pm 7.6^{\mathrm{b}}$ & $24.2 \pm 8.3^{c}$ \\
\hline $\mathrm{p}$-value $\S$ & $<0.00 I^{*}$ & $<0.00 I^{*}$ & $<0.00 I^{*}$ & $<0.00 I^{*}$ \\
\hline Normal & $27.9 \pm 12.2^{\mathrm{a}}$ & $76.9 \pm 9.0^{\mathrm{a}}$ & $1.0 \pm 1.6^{a}$ & $79.7 \pm 12.7^{\mathrm{a}}$ \\
\hline \multicolumn{5}{|l|}{ AGA } \\
\hline Ludwig I & $39.6 \pm 18.9^{b}$ & $64.5 \pm 11.0^{\mathrm{b}}$ & $9.2 \pm 7.2^{b}$ & $58.9 \pm 21.4^{b}$ \\
\hline Ludwig II & $48.9 \pm 19.9^{b}$ & $61.6 \pm 9.3^{b}$ & $7.1 \pm 7.3^{b}$ & $51.3 \pm 18.7^{b}$ \\
\hline p-value ${ }^{\S}$ & $<0.001 *$ & $<0.001 *$ & $<0.001 *$ & $<0.001 *$ \\
\hline
\end{tabular}

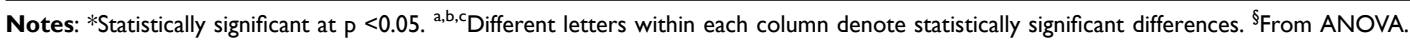

Abbreviations: AGA, androgenetic alopecia; TE, telogen effluvium.

For type III ( $48.7 \pm 23.8$ hairs), IV (60.5 \pm 34.3 hairs) according to the Hamilton-Norwood classification and type I (39.6 \pm 18.9 hairs) and II (48.9 \pm 19.9 hairs) according to the Ludwig scale of AGA patients, the number of hair loss were significantly higher than normal subjects $(27.9 \pm 12.2$ hairs, $\mathrm{p}<0.005)$. Other types of AGA were statistically no difference to the hair shedding numbers in normal subjects.

\section{Diameter of Shedding Hairs}

The results for hair diameter analysis are presented in Table 2 and Figure 2. Hair diameter in normal subjects was $76.9 \pm$ $9.0 \mu \mathrm{m}$. Patients diagnosed as AGA and TE yielded values of $60.0 \pm 9.5 \mu \mathrm{m}$ and $82.7 \pm 9.2 \mu \mathrm{m}$. There was a significant difference among AGA, TE and normal subjects $(\mathrm{p}<0.05)$.

For entire AGA cohort, all hair diameter values for different grades of MPHL and FPHL were significantly lower than those of normal subjects $(\mathrm{p}<0.001)$. The diameters were increasingly thinning as the severity of alopecia increased. However, there were no statistical differences within various degrees of AGA for either Hamilton-Norwood or Ludwig scales.

\section{Percentage of Vellus and Terminal Hairs}

The results for percentage of vellus and terminal hair analysis are presented in Table 2 and Figure 2. All the hairs were classified based on different diameters.

The percentage of vellus hairs in AGA patients was $8.3 \pm 6.6 \%$, which was significantly higher than that in either normal subjects or TE patients $(1.0 \pm 1.6 \%$ and $1.0 \pm 1.0 \%$, respectively; $\mathrm{p}<0.001)$. The values in all classifications of AGA were significantly higher than those in the normal group $(\mathrm{p}<0.05)$.

Concerning the percentage of terminal hairs, AGA group had a significantly lower value $(48.8 \pm 20.1 \%)$ compared with normal and TE groups $(79.7 \pm 12.7 \%$ and $85.3 \pm 8.7 \%$, respectively; $\mathrm{P}<0.001)$. The values in subjects with different 

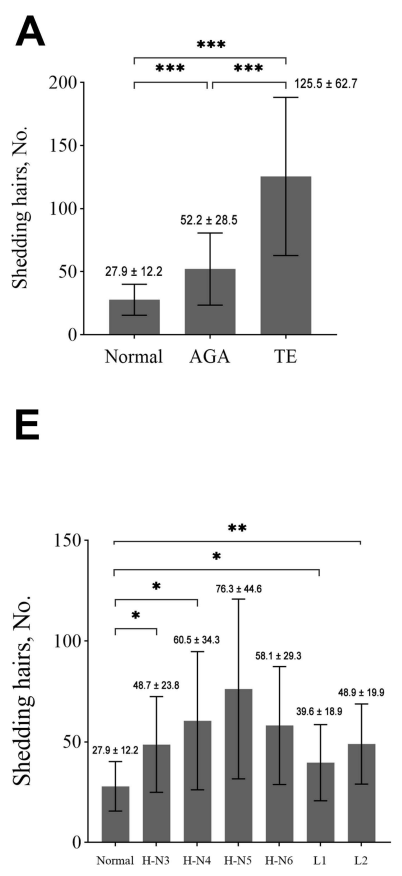

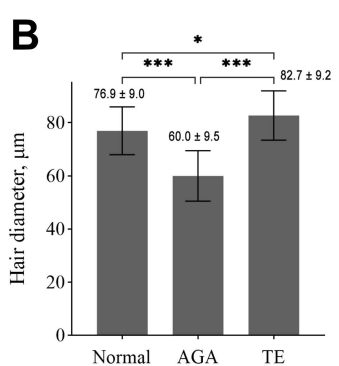

$\mathbf{F}$

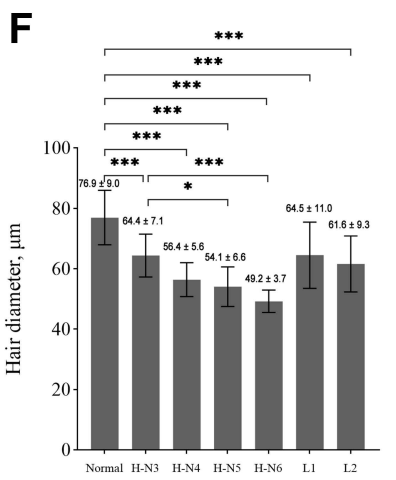

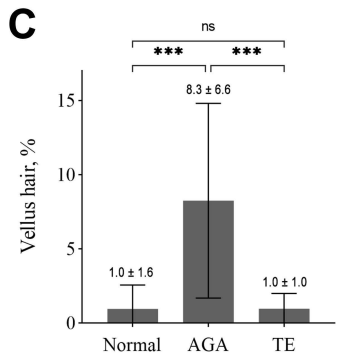

G

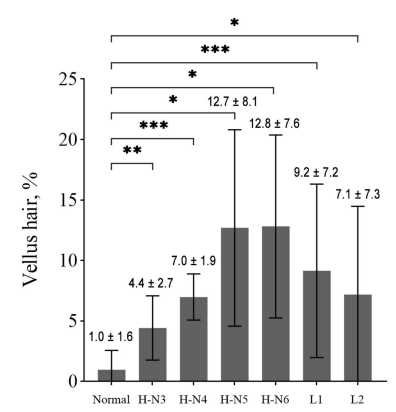

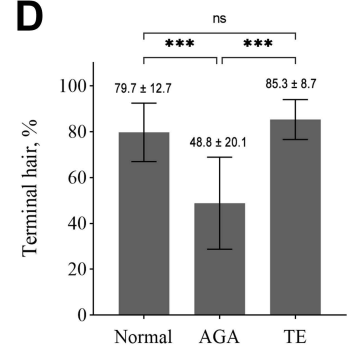

H

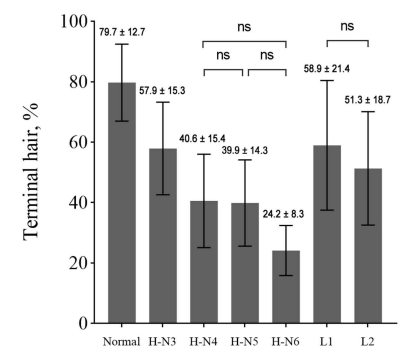

Figure 2 Comparisons of shed hair parameters in different groups. (A and $\mathbf{E}$ ) Shedding hair number. (B and $\mathbf{F})$ Hair diameter. (C and $\mathbf{G})$ Percentage of vellus hairs. (D and H) Percentage of terminal hairs.

Notes: *p $<0.05$. **p $<0.01$. ***p $<0.001$.

Abbreviations: AGA, androgenetic alopecia; TE, telogen effluvium; H-N, Hamilton-Norwood classification; L, Ludwig classification; MPHL, male pattern hair loss; FPHL, female pattern hair loss; ns, no significance.

types of AGA (including both MPHL and FPHL) were either significantly lower or tended to be lower with increasing severity degree of baldness and were all significantly lower than those of normal participants $(\mathrm{P}<0.001)$.

\section{Diagnostic Accuracy Using Shedding Hair Parameters}

Normal participants were taken as the reference to draw the ROC curves of shedding hair parameters for AGA and TE patients. The diagnostic performance of various parameters in different groups are presented in Table 3 and Figure 3. For AGA and normal groups, when used alone, shedding hair number and percentage of vellus hairs showed AUCs over $70 \%$, and the percentage of vellus hairs demonstrated higher diagnostic confidence compared with hair count (the areas under the AUC were 0.937 and 0.788, respectively; see Figure 3A). For the diagnosis of TE, shedding hair number alone showed significant correlation with TE patients. The ROC analysis confirmed an accuracy of 0.999 (95\% CI: 0.961-1.000) (Figure 3B). However, the percentage of vellus hairs or terminal hairs was not accurate in discriminating TE from normal subjects (AUCs approximately 0.500 and 0.622 , respectively; data not shown).

To distinguish between AGA and TE, shedding hair diameter had a higher AUC value of 0.963 (95\% CI: 0.932 0.994) than that of shedding hair number ( $\mathrm{AUC}=0.922,95 \% \mathrm{CI}: 0.872-0.971$ ) (Figure 3C). The approach of combination showed the most accuracy of 0.996 (95\% CI: $0.989-1.000)$.

\section{Discussion}

Trichoscopy is now the most used non-invasive technique available in clinical practice for the evaluation of hair loss disorders, allowing observation of hair shafts, follicle openings and perifollicular scalp skin alterations, as well as quantitative measurement of hair growth. ${ }^{12,16}$ When it comes to the shedding hairs, only the morphology of the hair shaft and hair root could be observed by trichoscopy; however, the number of shedding hairs could not be evaluated. Notably, shedding hair counts contain important information for diagnosis and treatment monitoring, and they are also the primary focus of patients' attention. Modified wash test has been introduced to collect and analyze shedding hairs for many years, and it has been shown that the information in shedding hairs could be valuable in diagnosing AGA and TE patients. ${ }^{10}$ 
Table 3 AUCs, Sensitivity, Specificity and Cut-off Value Using the Parameters of Shedding Hair Number, Percentage of Vellus Hairs and Combining the Two Markers as Suggested by ROC Curve Analysis Between the Groups of AGA and Normal Subjects, TE and Normal Subjects and AGA and TE Patients

\begin{tabular}{|c|c|c|c|c|c|c|}
\hline & Parameter & AUC $(95 \% \mathrm{Cl})$ & p-value & $\begin{array}{l}\text { Cut-off } \\
\text { Value }\end{array}$ & $\begin{array}{l}\text { Sensitivity } \\
(95 \% \mathrm{Cl})\end{array}$ & $\begin{array}{l}\text { Specificity } \\
(95 \% \mathrm{Cl})\end{array}$ \\
\hline \multirow{3}{*}{$\begin{array}{l}\text { AGA vs } \\
\text { Normal }\end{array}$} & Shedding hair number & $0.788(0.715-0.860)$ & 0.000 & 35.7 & $0.688(0.578-0.78 \mathrm{I})$ & $0.803(0.696-0.879)$ \\
\hline & $\begin{array}{l}\text { Percentage of vellus } \\
\text { hairs (\%) }\end{array}$ & $0.937(0.898-0.976)$ & 0.000 & 2.9 & $0.844(0.744-0.917)$ & $0.915(0.825-0.968)$ \\
\hline & $\begin{array}{l}\text { Combined double- } \\
\text { markers }\end{array}$ & $0.957(0.929-0.985)$ & 0.000 & & & \\
\hline \multirow[t]{3}{*}{ AGA vs TE } & Shedding hair number & $0.922(0.872-0.97 I)$ & 0.000 & 68.7 & $0.964(0.823-0.998)$ & $0.766(0.66 \mathrm{I}-0.847)$ \\
\hline & $\begin{array}{l}\text { Shedding hair diameter } \\
(\mathrm{mm})\end{array}$ & $0.963(0.932-0.994)$ & 0.000 & 69.9 & $0.964(0.823-0.998)$ & $0.870(0.777-0.928)$ \\
\hline & $\begin{array}{l}\text { Combined double- } \\
\text { markers }\end{array}$ & $0.996(0.989-1.000)$ & 0.000 & & & \\
\hline $\begin{array}{l}\text { TE vs } \\
\text { Normal }\end{array}$ & Shedding hair number & $0.999(0.96 \mathrm{I}-1.000)$ & 0.000 & 53.6 & $1.000(0.877-1.000)$ & $0.972(0.902-0.997)$ \\
\hline
\end{tabular}

Abbreviations: AGA, androgenetic alopecia; TE, telogen effluvium; $\mathrm{AUC}$, area under ROC curve; $\mathrm{Cl}$, confidence interval.

The result of wash test can be influenced by several factors, for instance: intervals of each hair wash, times of hair rinse during a hair wash procedure, manner of hair collection, the hairs collected during combing and drying right after the washing, combing times during the procedure, operation difficulty and compliance of patients. Thus, it is necessary to standardize the operating procedure and eliminate factors contributing to collection errors. Previously introduced wash tests are not widely used in clinical practice because of some procedural limitations, such as the hair length should be at least $3 \mathrm{~cm}$, and low compliance of subjects because of 5-day interval between two washings. RWT in this study might collect the shedding hairs more accurately because it fixes the shampoo time, shortens the shampoo interval (24 \pm 2 hours), limits the number of times for hair washing and combing, and collects all shedding hairs during the rinsing and drying processes. In our experience, all types of hair (terminal, intermediate and vellus hairs) of any length $(\geq 0.5 \mathrm{~cm}) \mathrm{can}$ be collected successfully by RWT.

There was no significant difference in all parameters of shedding hairs between 3-consecutive-days and 7-consecutive-days RWT. This study established an RWT method of 3 consecutive days, which was convenient for subjects to perform. In addition, a shorter duration of the test could also reduce probability of experiment interruptions and measurement workload. All participants showed high compliance and $100 \%$ of them finished the study.

In this study, shedding hair parameters in people without hair loss have been described. The average number of shedding hairs during daily washing was $27.9 \pm 12.2$ hairs, which was significantly lower than 100 hairs in theories, ${ }^{17}$ because numerous hairs may be lost in daily activities and escape from detection. In addition, we found the average

A

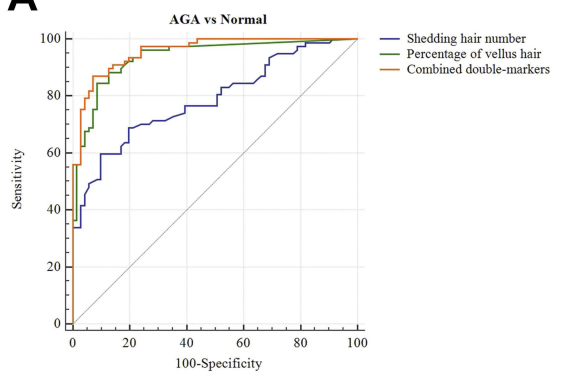

B

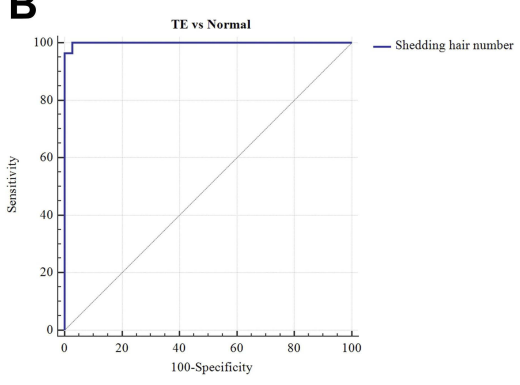

C

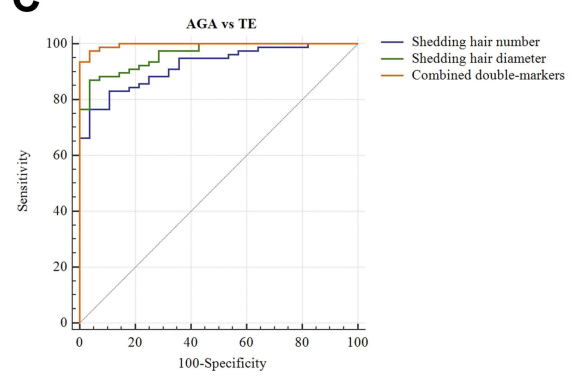

Figure 3 ROC curves of prediction of incidence between (A) AGA and normal groups, (B) TE and normal groups and (C) AGA and TE patients. Abbreviations: AGA, androgenetic alopecia; TE, telogen effluvium. 


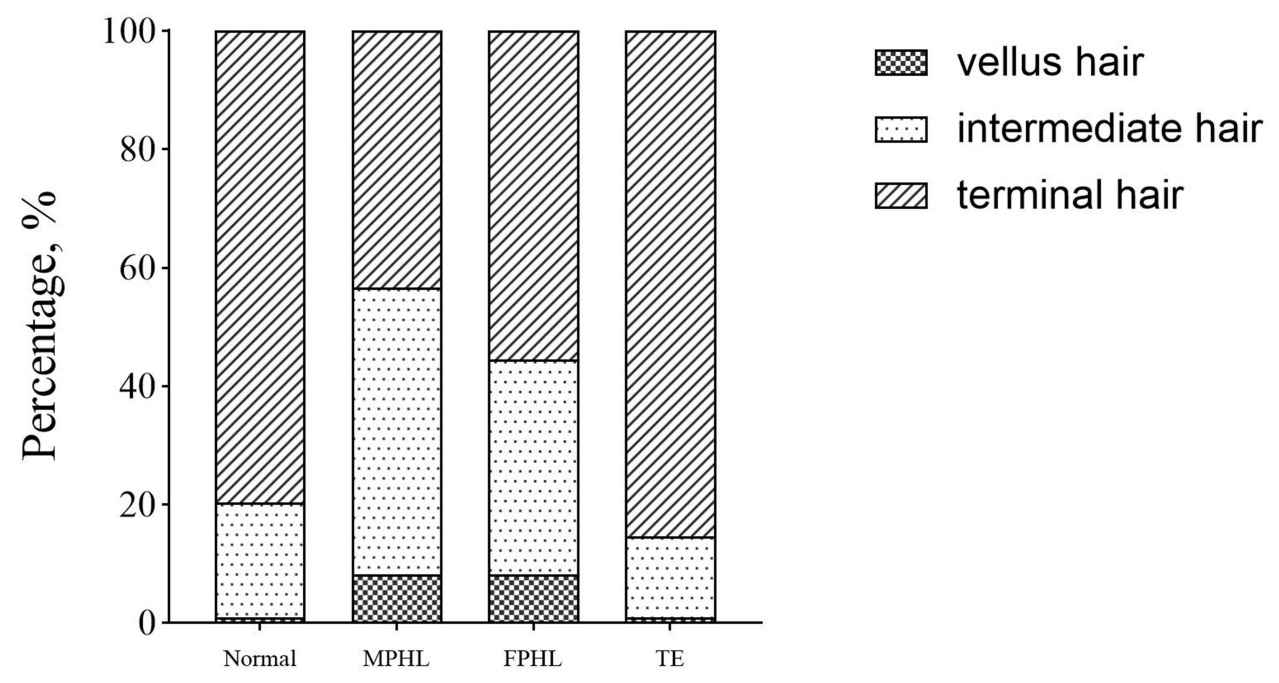

Figure 4 The proportion of shedding hair with different diameters.

Abbreviations: AGA, androgenetic alopecia; TE, telogen effluvium; MPHL, male pattern hair loss; FPHL, female pattern hair loss.

shedding hair diameter was $76.9 \pm 9.0 \mu \mathrm{m}$ in normal subjects. In Asia, the hair diameter has been studied on the scalp measured by non-invasive phototrichogram technique. The mean values in Chinese, Thai and Japanese were $89 \mu \mathrm{m}, 86.7$ $\mu \mathrm{m}$ and 79.3-87.1 $\mu \mathrm{m}$, respectively. ${ }^{18-20}$ Of note, shedding hair diameter in TE patients in this study $(82.7 \pm 9.2 \mu \mathrm{m})$ was closer to those. The possible reason is that vellus hairs were included and counted in this study, which might lower the average diameter of the hair shafts, while vellus hairs were not included for measurement in most of the previous studies. ${ }^{19,21}$ No significant difference was detected in shedding hair number or hair shaft diameter between males and females without hair loss.

Compared with previous studies of MWT, shedding hair counts of RWT were lower in AGA (52.2 \pm 28.5 vs $70.8 \pm$ 29.5 hairs) and TE patients (125.5 \pm 62.7 vs $247.9 \pm 187.1$ hairs) because the MWT needs a 5-consecutive-days astriction of hair wash before the test while RWT is a daily number. ${ }^{10}$ Shedding hair diameter, like that in normal group, also showed lower in the patients with AGA compared with that of the hair on the scalp (57.4 $\mu \mathrm{m}$ vs $58.5 \mu \mathrm{m}$ in MPHL, 63.2 $\mu \mathrm{m}$ vs $65.5-75.7 \mu \mathrm{m}$ in FPHL) ${ }^{22}$ In addition, we found that there was a significant difference in the percentage of vellus hairs between AGA and normal subjects. In normal individuals, $93.0 \%$ of them had no more than one vellus hair shed in a daily wash, which was remarkably less than in AGA patients (4.7 hairs on average). This suggests that people should be alert to the possibility of AGA and receive a further hair examination if they find several tiny thin hairs in the sink or bathtub during a shampoo process.

Interestingly, for AGA patients, female pattern alopecia showed lower intermediate hair and higher terminal hair proportions than male pattern alopecia (Figure 4). A similar distinction in the percentage of terminal hairs was also found by scalp punch biopsy. ${ }^{23}$ We hypothesize that the process of hair follicle miniaturization may be different in MPHL and FPHL. For MPHL, the miniaturization of follicles might occur gradually in a long time period over many hair cycles. In contrast, abrupt atrophy of follicles seemed to happen in FPHL patients within a shorter time, which might lead to fewer scalp intermediate hairs than MPHL patients. This distinction was also presented by Van Neste, ${ }^{24}$ however more studies are needed to confirm the hypotheses.

The number of shedding hairs increased with advancement of AGA severity, from grade III to V in MPHL and from grade L1 to L2 in FPHL. However, a surprisingly downward trend showed in the hair count increasing line in grade VI of MPHL. We hypothesize that in severe AGA (grade VI or higher of MPHL), the total hair follicle numbers on the scalp might have some degree of decrease. Interestingly, $\mathrm{Hu}$ et $\mathrm{al}^{25}$ observed that focal atrichia under trichoscopy were positively related to the severity of AGA and values were significantly increased in patients with type V to VII of MPHL. Early-stage AGA is more likely to be reversible; however, continuous progress of AGA eventually causes permanent, irreversible follicle loss. ${ }^{26}$ Treatment for AGA always works better when started early. ${ }^{27}$ Current available therapies are 
more helpful in stopping further progression of hair loss than in reversing vellus hair to terminal hair. ${ }^{28}$ Therefore, we suggest starting treatment for MPHL before progressing to grade VI.

TE is a diffuse non-scarring alopecia that occurs about $2-3$ months after an inciting event, which is characterized by a large proportion of anagen hairs stopping growing prematurely and subsequently entering catagen and then telogen phases. ${ }^{29}$ Shedding hair number always exceeds 100 , sometimes even more than 300 per day, and involves mainly terminal hairs. ${ }^{3,30}$ In this study with RWT test, the mean number of shedding hairs in TE patients was 125.5 hairs, which is about 4.5 times that of normal individuals and 2.4 times that of AGA patients. When the value is more than 53.6 hairs with normal vellus hair proportion, we could obtain a reasonably high sensitivity (100.0\%) and specificity (97.2\%) for the diagnosis of TE. In addition, repeat RWT every month can monitor the treatment effects in TE.

\section{Limitations}

The sample size of this study was relatively small and taking hair diameter measurements can be time-consuming. Further studies including more cases, particularly those with severe AGA (eg, grade VII of MPHL and grade III of FPHL), are needed. Moreover, optimizing the methods of hair counting and diameter measurement by artificial intelligence (AI) techniques based on portable dermoscopy of fixed magnifications may greatly reduce the workload. The current study provides a strong theoretical basis and preliminary data for future development of a modified version based on AI.

\section{Conclusion}

The RWT of 3 consecutive days allows for accurate assessment of daily shedding hairs during hair washing and diagnosis of AGA and TE. The RWT is a non-invasive, inexpensive, and easy-to-use tool for evaluating daily shedding hairs and monitoring treatment response and may increase patient compliance in dermatology clinics.

\section{Funding}

This study was supported by the National Natural Science Foundation of China (No. 81773311 and No. 82073459).

\section{Disclosure}

The authors report no conflicts of interest in this work.

\section{References}

1. Marks DH, Penzi LR, Ibler E, et al. The Medical and Psychosocial Associations of Alopecia: recognizing hair loss as more than a cosmetic concern. Am J Clin Dermatol. 2019;20(2):195-200. doi:10.1007/s40257-018-0405-2

2. Hadshiew IM, Foitzik K, Arck PC, et al. Burden of hair loss: stress and the underestimated psychosocial impact of telogen effluvium and androgenetic alopecia. J Invest Dermatol. 2004;123(3):455-457. doi:10.1111/j.0022-202X.2004.23237.x

3. Shapiro J. Clinical practice. Hair loss in women. N Engl J Med. 2007;357(16):1620-1630. doi:10.1056/NEJMcp072110

4. Werner B, Mulinari-Brenner F. Clinical and histological challenge in the differential diagnosis of diffuse alopecia: female androgenetic alopecia, telogen effluvium and alopecia areata - part I. An Bras Dermatol. 2012;87(5):742-747. doi:10.1590/S0365-05962012000500012

5. Dhurat R, Saraogi P. Hair evaluation methods: merits and demerits. Int J Trichology. 2009;1(2):108-119. doi:10.4103/0974-7753.58553

6. Olszewska M, Warszawik O, Rakowska A, et al. Methods of hair loss evaluation in patients with endocrine disorders. Endokrynol Pol. 2010;61 (4):406-411.

7. Rushton H, James KC, Mortimer CH. The unit area trichogram in the assessment of androgen-dependent alopecia. Br J Dermatol. 1983;109 (4):429-437. doi:10.1111/j.1365-2133.1983.tb04617.x

8. Sinclair R. Hair shedding in women: how much is too much? Br J Dermatol. 2015;173(3):846-848. doi:10.1111/bjd.13873

9. Rampini P, Guarrera M, Rampini E, et al. Assessing hair shedding in children. Dermatology. 1999;199(3):256-257. doi:10.1159/000018258

10. Rebora A, Guarrera M, Baldari M, et al. Distinguishing androgenetic alopecia from chronic telogen effluvium when associated in the same patient: a simple noninvasive method. Arch Dermatol. 2005;141(10):1243-1245. doi:10.1001/archderm.141.10.1243

11. Guarrera M, Cardo PP, Rebora A. Assessing the reliability of the Modified Wash Test. G Ital Dermatol Venereol. 2011;146(4):289-294.

12. Miteva M, Tosti A. Hair and scalp dermatoscopy. J Am Acad Dermatol. 2012;67(5):1040-1048. doi:10.1016/j.jaad.2012.02.013

13. Kerkemeyer KL, Trindade de Carvalho L, Jerjen R, et al. Female pattern hair loss in men: a distinct clinical variant of androgenetic alopecia. $J A m$ Acad Dermatol. 2021;85(1):260-262. doi:10.1016/j.jaad.2020.09.042

14. Mubki T, Rudnicka L, Olszewska M, et al. Evaluation and diagnosis of the hair loss patient: part I. History and clinical examination. $J$ Am Acad Dermatol. 2014;71(3):415.e1-415.e15. doi:10.1016/j.jaad.2014.04.070

15. Blume-Peytavi U. Hair Growth and Disorders. Springer Science \& Business Media; 2008. 
16. Leerunyakul K, Suchonwanit P. Evaluation of hair density and hair diameter in the adult Thai population using quantitative trichoscopic analysis. Biomed Res Int. 2020;2020:2476890. doi:10.1155/2020/2476890

17. Wasko CA, Mackley CL, Sperling LC, et al. Standardizing the 60-second hair count. Arch Dermatol. 2008;144(6):759-762. doi:10.1001/ archderm.144.6.759

18. Loussouarn G, Lozano I, Panhard S, et al. Diversity in human hair growth, diameter, colour and shape. An in vivo study on young adults from 24 different ethnic groups observed in the five continents. Eur J Dermatol. 2016;26(2):144-154. doi:10.1684/ejd.2015.2726

19. Rojhirunsakool S, Suchonwanit P. Parietal scalp is another affected area in female pattern hair loss: an analysis of hair density and hair diameter. Clin Cosmet Investig Dermatol. 2018;11:7-12. doi:10.2147/CCID.S153768

20. Tajima M, Hamada C, Arai T, et al. Characteristic features of Japanese women's hair with aging and with progressing hair loss. J Dermatol Sci. 2007;45(2):93-103. doi:10.1016/j.jdermsci.2006.10.011

21. Lopez V, Martin JM, Sanchez R, et al. Usefulness of TrichoScan professional in the evaluation of hair loss in females. Report of 180 cases. $J$ Eur Acad Dermatol Venereol. 2011;25(9):1068-1072. doi:10.1111/j.1468-3083.2010.03924.x

22. Kang H, Kang TW, Lee SD, et al. The changing patterns of hair density and thickness in South Korean women with hair loss: clinical office-based phototrichogram analysis. Int J Dermatol. 2009;48(1):14-21. doi:10.1111/j.1365-4632.2009.03795.x

23. Whiting DA, Waldstreicher J, Sanchez M, et al. Measuring reversal of hair miniaturization in androgenetic alopecia by follicular counts in horizontal sections of serial scalp biopsies: results of finasteride $1 \mathrm{mg}$ treatment of men and postmenopausal women. J Investig Dermatol Symp Proc. 1999;4(3):282-284. doi:10.1038/sj.jidsp.5640230

24. Van Neste D. Female patients complaining about hair loss: documentation of defective scalp hair dynamics with contrast-enhanced phototrichogram. Skin Res Technol. 2006;12(2):83-88. doi:10.1111/j.0909-752X.2006.00202.x

25. Hu R, Xu F, Han Y, et al. Trichoscopic findings of androgenetic alopecia and their association with disease severity. J Dermatol. 2015;42 (6):602-607. doi:10.1111/1346-8138.12857

26. Messenger AG, Sinclair R. Follicular miniaturization in female pattern hair loss: clinicopathological correlations. Br $J$ Dermatol. 2006; 155 (5):926-930. doi:10.1111/j.1365-2133.2006.07409.x

27. Rossi A, Anzalone A, Fortuna MC, et al. Multi-therapies in androgenetic alopecia: review and clinical experiences. Dermatol Ther. 2016;29 (6):424-432. doi:10.1111/dth.12390

28. Hugh Rushton D, Norris MJ, Van Neste D. Hair regrowth in male and female pattern hair loss does not involve the conversion of vellus hair to terminal hair. Exp Dermatol. 2016;25(6):482-484. doi:10.1111/exd.12945

29. Chien Yin GO, Siong-See JL, Wang ECE. Telogen Effluvium - A review of the science and current obstacles. J Dermatol Sci. 2021;101 (3):156-163. doi:10.1016/j.jdermsci.2021.01.007

30. Hughes EC, Saleh D. Telogen Effluvium. StatPearls; 2020.

Clinical, Cosmetic and Investigational Dermatology

Dovepress

Publish your work in this journal

Clinical, Cosmetic and Investigational Dermatology is an international, peer-reviewed, open access, online journal that focuses on the latest clinical and experimental research in all aspects of skin disease and cosmetic interventions. This journal is indexed on CAS. The manuscript management system is completely online and includes a very quick and fair peer-review system, which is all easy to use. Visit http://www. dovepress.com/testimonials.php to read real quotes from published authors.

Submit your manuscript here: https://www.dovepress.com/clinical-cosmetic-and-investigational-dermatology-journal 